\title{
BMJ Open Psychological impact of repeated epidemic exposure on healthcare workers: findings from an online survey of a healthcare workforce exposed to both SARS (severe acute respiratory syndrome) and COVID-19
}

\author{
Lai Gwen Chan (D) , ${ }^{1}$ Pei Lin Lynnette Tan, ${ }^{1}$ Kang Sim (D) ,, ${ }^{2}$ Ming Yee Tan, ${ }^{3}$ \\ Kah Hong Goh, ${ }^{4}$ Pei Qi Su, ${ }^{5}$ Alvin Kah Heng Tan, ${ }^{6}$ Eng Sing Lee (1) , ${ }^{7}$ Shu Yun Tan, ${ }^{7}$ \\ Wen Phei Lim, ${ }^{8}$ Chia Hui Aw, ${ }^{9}$ Yi Zhen Goh, ${ }^{10}$ Sapna Sadarangani, ${ }^{11,12}$ \\ Angela Chow ${ }^{13,14}$
}

To cite: Chan LG, Tan PLL, Sim K, et al. Psychological impact of repeated epidemic exposure on healthcare workers: findings from an online survey of a healthcare workforce exposed to both SARS (severe acute respiratory syndrome) and COVID-19. BMJ Open 2021;11:e051895. doi:10.1136/ bmjopen-2021-051895

- Prepublication history and additional supplemental material for this paper are available online. To view these files, please visit the journal online (http://dx.doi.org/10.1136/ bmjopen-2021-051895)

Received 23 April 2021 Accepted 09 November 2021

Check for updates

(c) Author(s) (or their employer(s)) 2021. Re-use permitted under CC BY-NC. No commercial re-use. See rights and permissions. Published by BMJ.

For numbered affiliations see end of article.

Correspondence to

Dr Lai Gwen Chan;

lai_gwen_chan@ttsh.com.sg

\section{ABSTRACT}

Objective To measure the psychological well-being of healthcare workers (HCWs) during this COVID-19 pandemic and examine the experiences of the subgroup of participants who were also HCWs during the 2003 SARS epidemic.

Design Anonymous online survey adapted from a similar study conducted during the SARS epidemic, disseminated from July 2020 to August 2020.

Setting Nine healthcare institutions across Singapore ranging from primary care, community care, tertiary care and specialised referral centres.

Participants Employees working in the participating healthcare institutions.

Results of 3828 survey returns, 3616 had at least one completed item on the questionnaire. Majority were female (74.7\%), nurses (51.7\%), foreign-born (53.2\%) and not working in the tertiary care setting $(52.1 \%)$. The median score on the Impact of Events Scale (IES) was 15 (IQR 23) and $28.2 \%$ of the sample scored in the moderate/severe range. $22.7 \%$ of the participants were also HCWs during SARS and more than half of them felt safer and better equipped in the current pandemic. $25.2 \%$ of SARS HCWs and $25.9 \%$ of non-SARS HCWs had moderate/severe IES scores $(p=0.904)$. After adjusting for age, marital status, parity and length of work experience, racial minority groups and living apart from family were independent predictors of high IES regardless of prior SARS epidemic experience. Daily exposure to confirmed or suspect COVID-19 cases increased the odds of high IES for nonSARS HCWs only.

Conclusions and relevance Overall, while $28 \%$ of HCWs in our study suffered from significant trauma-related psychological symptoms regardless of prior experience with the SARS epidemic, those with prior experience reported feeling safer and better equipped, finding the workload easier to manage, as well as having more confidence in their healthcare leaders. We recommend for more trauma-informed support strategies for our HCWs
Strengths and limitations of this study

- A strength of this study is its novelty and the future applications of the findings to aid the mental resilience and wellness of healthcare workers exposed to multiple healthcare crises.

- Another strength of the study is its large and diverse cohort spanning nine healthcare institutes ranging from primary to tertiary care in Singapore, which allows for good external validity within the country's healthcare system.

- Additionally, the questionnaire was directly developed from a previous study on healthcare workers in Singapore during the SARS epidemic, allowing for direct comparison of the two healthcare crises.

- One limitation of study was that the Impact of Events Scale-Revised scale and other scales that measured depression/anxiety levels, personality and resilience factors were not used, reducing the comparability of the results with those from other studies.

especially those from racial minority groups, who are foreign-born and isolated from their families.

\section{INTRODUCTION}

A major healthcare challenge of our time is undoubtedly emerging infectious disease epidemics and pandemics. Healthcare workers (HCWs) are indispensable in the response to such crises and are hence placed in a position of medical and psychological risks. Protecting HCWs from such risks and enhancing their resilience is vital in keeping healthcare systems robust and intact for effective crisis response. 
Singapore is no stranger to such crises. One of the most well documented is that of the 2003 SARS epidemic that lasted from 12 March to 4 June, resulting in 238 cases ( $42 \%$ of which were HCWs) and 33 deaths, of which five were HCWs. Towards the end of that epidemic, Koh et al studied risk perception and impact on personal and work lives of 15000 HCWs from nine healthcare institutions in Singapore, and found that a majority perceived great personal risk of falling ill but accepted it, and that more than half experienced increased anxiety, work stress, workload, and social stigmatisation. ${ }^{1}$ Another single-site local study by Chan and Huak found that $27 \%$ of HCWs surveyed had significant emotional distress with $20 \%$ reporting the possible presence of post-traumatic stress disorder. ${ }^{2}$ A similar study in Hong Kong repeated psychological measures among HCWs 1 year after the peak of the epidemic and found that HCWs from high-risk areas had higher and sustained levels of perceived stress that was associated with higher levels of depression, anxiety and post-traumatic stress as compared with HCWs from lowrisk areas. ${ }^{3}$ This and several other studies from localities similarly affected by $\mathrm{SARS}^{4-7}$ identified risk factors such as job stress, social isolation and maladaptive coping; and protective factors such as strong social support, perceived adequacy of training and positive coping styles, which led to the development of staff training and psychological wellness plans for outbreak preparedness.

All hospitals implemented mandatory structured training for all front-line HCWs in the use of personal protective equipment (PPE) with documentation of N95 mask fitting in employee records. Peer support programmes which included crisis hotlines for staff and access to early intervention were also implemented in each hospital. Post-SARS, Singapore had also purposefully built a National Centre for Infectious Diseases (NCID) to strengthen the country's capabilities in infectious disease management and prevention that became operational in May 2019. All these were put to the test during the current COVID-19 pandemic. A systematic review and meta-analysis of studies reporting psychological symptoms among HCWs at the front line of this pandemic published up to April 2020 showed that almost a quarter of HCWs surveyed exhibited depressive and anxious symptoms, and insomnia was prevalent in almost $40 \%{ }^{8}$

Healthcare in Singapore relies heavily on foreign HCW with about a third of the nurses being foreign nationals who hold temporary worker status. This study aimed to examine the psychological well-being and risk perception of the local HCW population during the COVID-19 pandemic. It was adapted from Koh et als survey done in 2003 to enable a general comparison between the two survey cohorts. ${ }^{1}$ This study also aimed to examine the experiences of the COVID-19 pandemic as compared with the SARS epidemic among a subgroup of respondents who were also working in healthcare during the 2003 SARS epidemic, hypothesising that HCWs who had experienced the SARS outbreak would be more resilient. We believe that this is the first study with such an aim and methodology. We hope to identify characteristics of both distressed and non-distressed HCWs who were also involved in Singapore's response to the SARS epidemic; this may help to inform strategies to boost the resilience of HCWs and prevent burnout and other psychological and psychiatric morbidity.

\section{METHODS}

Nine major healthcare institutions spanning from primary care, intermediate and long-term care (ILTC) to tertiary care participated in the anonymous, online survey that was disseminated by email blasts, recruitment posters with $\mathrm{QR}$ codes linked to the survey, and secure workplace messaging systems from mid-July to mid-August 2020. This was about 6 months after the first confirmed case in Singapore and about 1 month after the gradual cessation of the nationwide 'Circuit Breaker' lockdown period. ${ }^{9}$ These included Tan Tock Seng Hospital (which includes the colocated NCID that bore the brunt of the nation's COVID-19 adult patient load), Khoo Teck Puat Hospital, Institute of Mental Health, $\mathrm{Ng}$ Teng Fong General Hospital, Woodlands Health Campus (a new hospital that had not begun operating and whose staff were nested in other institutions), National Skin Centre, Dover Park Hospice, Community Care partners supported by the Agency for Integrated Care, National Healthcare Group Polyclinics and their general practitioner partners. Significant differences from participants of the 2003 study were the participation of the country's only psychiatric institution, more ILTC institutions and Woodlands Health Campus. Dental clinics that were part of the survey sample in 2003 were not included in this study.

Similar to the 2003 survey, our survey comprised three parts: (1) individual characteristics; (2) questions pertaining to the perception of exposure to COVID-19, perceived risk of infection and impact of the outbreak on personal and work life; and (3) Impact of Events Scale (IES) which measures subjective distress in response to stressful events (online supplemental files 1 and 2). There were several differences in the current survey for contextual accuracy. In the first section, we included questions asking respondents on their sites of deployment and scope of work because the rapid siting of cases in temporary community facilities staffed by deployed manpower was a major part of the pandemic response. In a novel section specific to those who were HCWs during the SARS epidemic, we surveyed participants' job titles, scope of work and exposure then, and measured their sense of safety, stress, preparedness, workload, and confidence in leadership now as compared with 2003 on a 7-point Likert scale. In the second section, we included new questions eliciting level of agreement on a 7-point Likert scale with measures specific to the current pandemic such as safe distancing, mandatory twice-daily logging of temperature, and use of a national contact tracing mobile application (TraceTogether). Under the section of impact on personal lives, we included questions about excessive searching of the internet for information and the impact of social media posts, and whether they or their personal contacts had been suspected of having or were confirmed to have COVID-19. Additionally, we sought their 
levels of agreement with other implemented public health measures. For the IES, we computed the mean and median scores, and the proportion of respondents who scored in the moderate/severe score range $(>25) .^{10}$

Next, we compared the subgroups of those who had also been HCWs during the SARS epidemic (SARS HCW) against the group of HCWs who had not (non-SARS HCW) on various measures, using $\chi^{2}$ tests for categorical variables and Student's t-test for quantitative variables. Univariate logistic regression was used to determine predictors of high IES scores, followed by multivariate logistic regression to adjust for confounders.

We analysed the data using STATA V.11.0 (released 2009) (online supplemental file 3 ).

\section{Patient and public involvement}

Patients and/or the public were not involved in the design, or conduct, or reporting, or dissemination plans of our research.

\section{RESULTS}

Out of 3828 returns of the survey, 3616 had completed at least 1 item (online supplemental file 4 ). Table 1 shows the demographic characteristics of the entire study sample. Majority of the respondents were female $(74.5 \%)$, aged $31-50$ years $(57.5 \%)$, nurses $(51.7 \%)$ with 10 or fewer years of working experience $(56.1 \%)$ and foreign-born $(53.2 \%)$. Most were married $(52.9 \%)$ with no children

\begin{tabular}{|c|c|c|c|c|c|}
\hline Characteristic $(\mathrm{N}=3616)$ & $\mathbf{n}$ & $\%$ & Characteristic $(\mathrm{N}=3616)$ & $\mathbf{n}$ & $\%$ \\
\hline Gender & & & Staying with & & \\
\hline Male & 916 & 25.3 & Family & 2183 & 60.4 \\
\hline Female & 2700 & 74.7 & Alone & 214 & 5.9 \\
\hline Age group & & & Hostel & 128 & 3.5 \\
\hline $21-30$ & 1038 & 28.7 & Rented room & 1080 & 29.9 \\
\hline $31-40$ & 1344 & 37.2 & Length of work experience & & \\
\hline $41-50$ & 735 & 20.3 & $0-10$ years & 2029 & 56.1 \\
\hline $51-60$ & 344 & 9.5 & $11-20$ years & 1050 & 29.0 \\
\hline$>60$ & 150 & 4.2 & $21-30$ years & 294 & 8.1 \\
\hline Ethnic group & & & $>30$ years & 210 & 5.8 \\
\hline Chinese & 1656 & 45.8 & Job title & & \\
\hline Malay & 327 & 9.0 & Doctor & 305 & 8.4 \\
\hline Indian & 553 & 15.3 & Nurse & 1870 & 51.7 \\
\hline Filipino & 627 & 17.3 & Allied health & 677 & 18.7 \\
\hline Others & 453 & 12.5 & Administrative etc & 739 & 20.4 \\
\hline Birth country & & & Site of work/deployment & & \\
\hline Singapore & 1694 & 46.9 & Acute/tertiary hospital & 1733 & 47.9 \\
\hline Malaysia & 387 & 10.7 & Dormitory/screening centre & 366 & 10.1 \\
\hline Philippines & 627 & 17.3 & Community care facility & 102 & 2.8 \\
\hline China & 154 & 4.3 & Primary care & 334 & 9.2 \\
\hline India & 286 & 7.9 & Intermediate/long-term care & 827 & 22.9 \\
\hline Myanmar and others & 468 & 12.9 & None of the above & 474 & 13.1 \\
\hline Marital status & & & $\begin{array}{l}\text { Daily exposure to confirmed or } \\
\text { suspect COVID- } 19 \text { patients }\end{array}$ & 404 & 11.2 \\
\hline Single & 1564 & 43.3 & Was a HCW during SARS & & \\
\hline Married & 1911 & 52.9 & Yes & 789 & 21.8 \\
\hline Divorced/widowed & 130 & 3.6 & No & 2690 & 74.4 \\
\hline No of children & & & IES score & & \\
\hline 0 & 1959 & 54.2 & Mean & 17.74 & (SD 14.7 \\
\hline $1-2$ & 1256 & 34.7 & Median & 15 & (IQR 23) \\
\hline Three or more & 390 & 10.8 & Moderate/severe range & 896 & 28.2 \\
\hline
\end{tabular}

$(\mathrm{N}=3616)$.

HCW, healthcare worker; IES, Impact of Events Scale. 
$(54.2 \%)$ and living with their families $(60.4 \%)$. Slightly more than $10 \%$ reported daily exposure to confirmed or suspect COVID-19 cases in their work. About a fifth $(21.8 \%)$ were also HCWs during the SARS outbreak. The overall mean IES score for the sample was 17.7 (SD 14.7) and the median score was subclinical (15, IQR 23), but more than a quarter $(28.2 \%)$ scored in the moderate/ severe range (total score $>25$ ).

Comparing the SARS HCWs and the non-SARS HCWs (table 2), the SARS-experienced group was significantly more likely to be older, to be local-born, married with children and staying with family, with longer working

\begin{tabular}{|c|c|c|c|c|c|c|c|}
\hline $\begin{array}{l}\text { Characteristic } \\
(\mathrm{N}=3479)\end{array}$ & $\begin{array}{l}\text { SARS, } \\
n=789(\%)\end{array}$ & $\begin{array}{l}\text { Non-SARS, } \\
\mathrm{n}=2690(\%)\end{array}$ & $P$ value & $\begin{array}{l}\text { Characteristic } \\
(\mathrm{N}=3479)\end{array}$ & $\begin{array}{l}\text { SARS, } n=789 \\
(\%)\end{array}$ & $\begin{array}{l}\text { Non-SARS, } \\
\mathrm{n}=2690(\%)\end{array}$ & $P$ value \\
\hline Male & 25.9 & 25.5 & 0.841 & Family & 69.3 & 57.8 & $<0.001$ \\
\hline Female & 74.1 & 74.5 & & Alone & 8.1 & 5.2 & \\
\hline $21-30$ & 3.8 & 35.7 & $<0.001$ & Rented room & 20.4 & 33.1 & \\
\hline $31-40$ & 12.9 & 44.5 & & \multicolumn{2}{|c|}{ Length of work experience } & & \\
\hline $41-50$ & 44.7 & 13.4 & & $0-10$ years & 8.5 & 70.1 & $<0.001$ \\
\hline $51-60$ & 24.8 & 4.9 & & $11-20$ years & 38.3 & 27.1 & \\
\hline$>60$ & 13.7 & 1.4 & & $21-30$ years & 29.4 & 2.1 & \\
\hline Indian & 16.1 & 15.2 & & Nurse & 59.7 & 50.3 & \\
\hline Filipino & 14.2 & 18.5 & & Allied health & 15.5 & 19.8 & \\
\hline Others & 16.2 & 11.6 & & $\begin{array}{l}\text { Administrative } \\
\text { etc }\end{array}$ & 11.7 & 22.6 & \\
\hline Birth country & & & & \multicolumn{2}{|c|}{ Site of work/deployment } & & \\
\hline Singapore & 51.2 & 45.1 & $<0.001$ & $\begin{array}{l}\text { Acute/tertiary } \\
\text { hospital }\end{array}$ & 52.7 & 48.6 & 0.694 \\
\hline Malaysia & 7.2 & 11.6 & & $\begin{array}{l}\text { Dormitory/ } \\
\text { screening centre }\end{array}$ & 9.5 & 10.7 & \\
\hline \multirow{4}{*}{$\begin{array}{l}\text { Marital status } \\
\text { Single } \\
\text { Married } \\
\text { Divorced/widowed }\end{array}$} & & & & \multirow{4}{*}{$\begin{array}{l}\text { Daily exposure } \\
\text { to confirmed or } \\
\text { suspect COVID-19 } \\
\text { patients }\end{array}$} & \multirow[t]{4}{*}{14.8} & \multirow[t]{4}{*}{10.7} & \multirow[t]{4}{*}{0.001} \\
\hline & 24.6 & 48.5 & $<0.001$ & & & & \\
\hline & 70.0 & 48.6 & & & & & \\
\hline & 5.5 & 2.9 & & & & & \\
\hline No of children & & & & IES score & & & \\
\hline 0 & 36.0 & 59.4 & $<0.001$ & Mean & $\begin{array}{l}16.7 \\
(S D 14.7)\end{array}$ & $\begin{array}{l}18.0 \\
(S D 14.8)\end{array}$ & 0.032 \\
\hline $1-2$ & 47.3 & 31.4 & & Median & $\begin{array}{l}13 \\
\text { (IQR 23) }\end{array}$ & $\begin{array}{l}15 \\
\text { (IQR 22) }\end{array}$ & 0.016 \\
\hline Three or more & 16.7 & 9.2 & & $\begin{array}{l}\text { Moderate/severe } \\
\text { range (\%) }\end{array}$ & 25.2 & 25.9 & 0.904 \\
\hline
\end{tabular}

HCW, healthcare worker; IES, Impact of Events Scale. 
experience. This is likely because many of the foreign HCWs during SARS would have left the country in the interim due to non-renewal of the work passes. There were significantly more respondents who were doctors in the SARS group. There was no difference in the proportion of respondents in both groups who had clinically significant IES scores (total $>25$ ) (25.2\% vs $25.9 \%, \mathrm{p}=0.904$ ). Their mean and median IES scores were not significantly different and did not reach clinical threshold.

Furthermore, more than half of the SARS HCWs rated that they felt safer $(54.8 \%)$, better equipped $(72.1 \%)$ with better managed workload $(58.9 \%)$ in the current pandemic as compared with during the SARS epidemic. Seventy-seven per cent felt that the authorities and healthcare leaders were doing a better job now as compared with before. However, only slightly less than half $(47.3 \%)$ felt less stressed now as compared with their past SARS experience (table 3 ).

\section{Regarding personal risk perception}

Both SARS HCWs and non-SARS HCWs did not differ in that approximately half of each group felt that their job put them at great risk of contracting COVID-19 and about $63 \%$ of both groups were afraid of contracting the disease (table 3). Despite this fear, both groups also did not differ in that only a small proportion felt that they should not be looking after COVID-19 patients $(14.0 \%$ vs $13.2 \%$, $\mathrm{p}=0.572)$ or were thinking of leaving their jobs $(5.4 \%$ vs $5.0 \%, \mathrm{p}=0.685$ ). However, significantly more non-SARS HCWs felt that they had little control over whether they would get infected ( $36.0 \%$ vs $46.2 \%$, $\mathrm{p}<0.001)$.

\section{Regarding the usefulness of protective measures}

SARS HCWs showed higher rates of disagreement with the protectiveness of standard PPE $(9.8 \%$ vs $6.5 \%$, $\mathrm{p}=0.003)$ and the powered air-purifying respirator $(22.8 \%$ vs $16.1 \%, \mathrm{p}<0.001$ ), though the majority of both groups disagreed that the current PPE they were provided with was inadequate $(71.0 \%$ vs $68.9 \%, p=0.28)$ (table 3 ). However, the SARS HCWs also showed lower rates of disagreement with the use of the TraceTogether $(20.0 \%$ vs $25.5 \%, \mathrm{p}=0.003)$. Non-SARS HCWs showed lower rates of disagreement with the items: being satisfied with the explanations for the various protective measures $(10.8 \%$ vs $14.9 \%, \mathrm{p}=0.005)$, having someone to turn to when they have a problem using PPE (10.2\% vs $16.3 \%, \mathrm{p}<0.001)$, that emotional support was available $(15.1 \%$ vs $20.4 \%$, $\mathrm{p}=0.001)$, and that clear policies and protocols were instituted $(10.8 \%$ vs $15.0 \%, p=0.004)$. Significantly more of the SARS HCWs also disagreed with the statement that they still found it difficult to follow the policies and protocols despite the help provided ( $77.5 \%$ vs $72.5 \%, \mathrm{p}=0.007)$.

\section{Regarding impact on personal life and work}

With respect to stigma, SARS HCWs showed higher rates of agreement with feeling appreciated by their employing institution $(74.7 \%$ vs $67.0 \%, \mathrm{p}<0.001)$ and feeling appreciated by society $(70.5 \%$ vs $62.5 \%, \mathrm{p}<0.001)$ (table 3). More of this group also agreed that there were adequate staff at work to handle the demands $(62.8 \%$ vs $55.3 \%, \mathrm{p}<0.001)$. There were relatively high numbers from both groups who reported: more conflict among work colleagues $(\sim 25 \%)$, feeling more stressed at work $(\sim 45 \%)$, having an increased workload $(\sim 58 \%)$, having to work overtime $(\sim 35 \%)$, having to do what they normally do not $(\sim 60 \%)$, spending more time on the internet searching for COVID-19 information ( 43\%), and that too much COVID-19 news on social media has affected them $(\sim 36 \%)$, with no significant difference on these items.

The comparison of SARS and non-SARS HCWs who scored in the moderate/severe range of the IES is shown in table 4. Variables that showed statistically significant differences between both groups were entered into univariate logistic regression, followed by multivariable logistic regression.

Table 5 shows the identified predictor variables for clinically significant IES scores in the different exposure groups.

For SARS HCWs, multivariate logistic regression showed that those who were non-Chinese (Indian, adjusted OR 2.33, SE $0.73,95 \%$ CI 1.26 to $4.32, \mathrm{p}=0.012$ ) and currently not living with family (Rented room, adjusted OR 2.45, SE $0.69,95 \%$ CI 1.41 to $4.26, \mathrm{p}=0.003$ ) were more than twice as likely to be significantly stressed by the current pandemic (Likelihood Ratio $\chi^{2}=64.74$, df 21, $\mathrm{p}=0.00$ ). For the non-SARS HCWs, those living apart from family (Rented room, adjusted OR 2.01, SE $0.30,95 \%$ CI 1.50 to $2.70, \mathrm{p}<0.001$ ) were two times more likely to be significantly stressed (Likelihood ratio $\chi^{2}=103.7$, df 21, $\mathrm{p}=0.00$ ). Additionally, those coming into daily exposure of confirmed/suspect COVID-19 cases were also 1.5 times more likely to experience significant stress (adjusted OR $1.53,95 \%$ CI 1.16 to 2.02, $\mathrm{p}=0.002$ ). Foreign-born participants were just as likely to be significantly stressed as compared with the local Singaporeans in either group.

\section{DISCUSSION}

We found that $28 \%$ of HCWs regardless of prior experience of the SARS epidemic had clinically significant IES scores that indicated significant post-traumatic symptoms. For SARS HCWs, this was higher than the $20 \%$ reported from a local non-designated SARS hospital during the SARS epidemic in 2003. ${ }^{2}$ Additionally, this proportion was not lower than the non-SARS HCWs group as we expected. One reason for this could be the larger scale impact and longer duration of COVID-19 compared with SARS. This could also indicate potential cumulative effects of repeated exposure to front-line trauma stemming from an epidemic/pandemic response in increasing the risk of developing clinically significant psychological symptoms instead of increasing one's resilience and protection from such effects. ${ }^{11}$ Another possible reason for this result could be that SARS HCWs were already experiencing persistent symptoms post-SARS and these effects 
Table 3 Comparison of personal risk perception and impact on personal and work lives between SARS and non-SARS HCWs

\begin{tabular}{|c|c|c|c|c|}
\hline Statement & $\begin{array}{l}\text { SARS HCW } \\
(n=748)\end{array}$ & $\begin{array}{l}\text { Non-SARS HCW } \\
(\mathrm{n}=2591)\end{array}$ & $P$ value & Effect size $\varphi$ \\
\hline Comparison with SARS experience & $\%$ agree & & & \\
\hline I feel less stressed now compared with SARS & 47.3 & & & \\
\hline I feel better equipped now compared with SARS & 72.1 & & & \\
\hline $\begin{array}{l}\text { I feel my workload now is better managed compared with during } \\
\text { SARS }\end{array}$ & 58.9 & & & \\
\hline
\end{tabular}

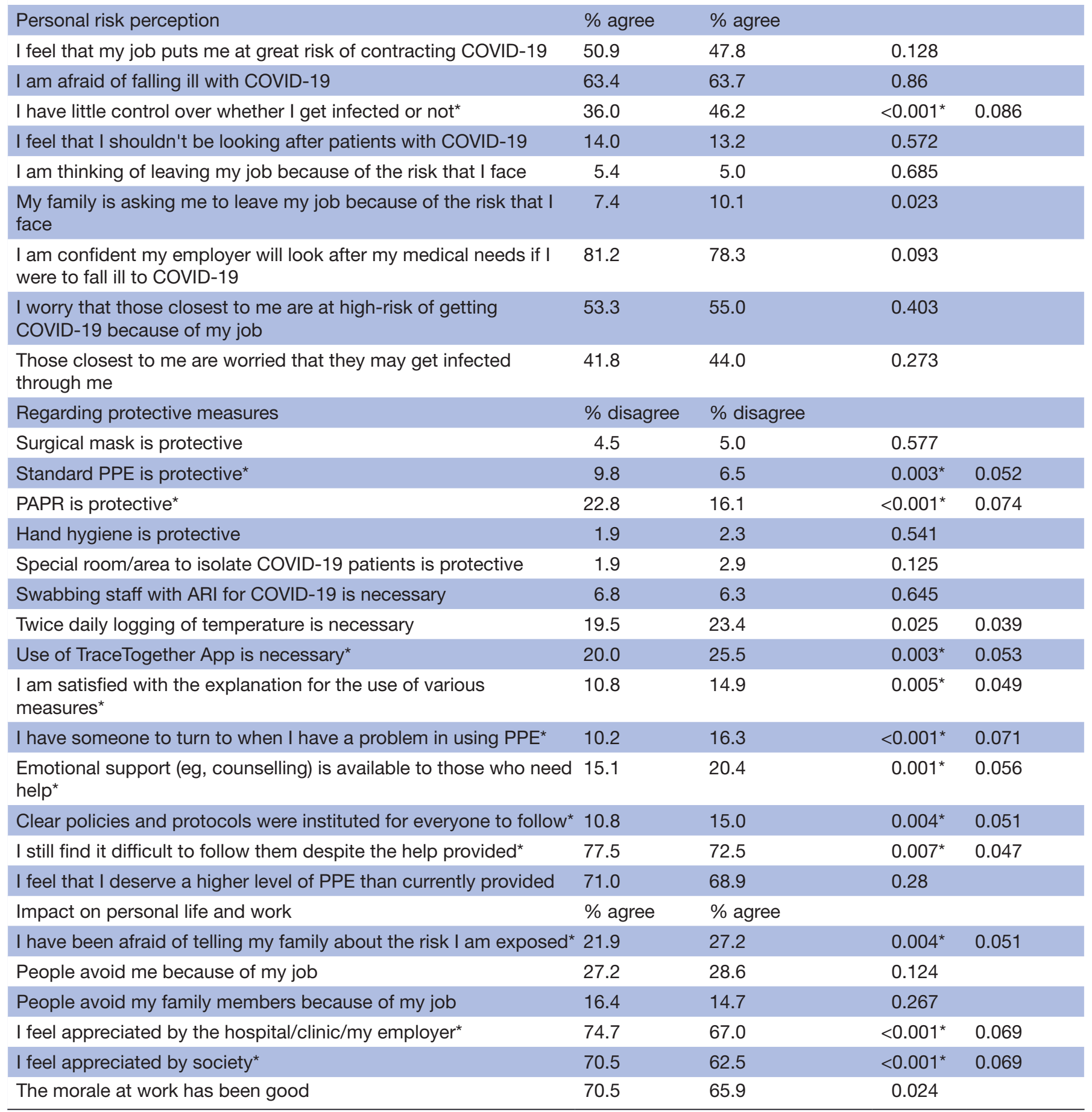


Table 3 Continued

\begin{tabular}{|c|c|c|c|c|}
\hline Statement & $\begin{array}{l}\text { SARS HCW } \\
(\mathrm{n}=748)\end{array}$ & $\begin{array}{l}\text { Non-SARS HCW } \\
(\mathrm{n}=2591)\end{array}$ & $P$ value & Effect size $\varphi$ \\
\hline $\begin{array}{l}\text { There are adequate staff at my workplace to handle the different } \\
\text { demands* }\end{array}$ & 62.8 & 55.3 & $<0.001^{*}$ & 0.063 \\
\hline There is more conflict among colleagues at work & 24.0 & 24.5 & 0.747 & \\
\hline I feel more stressed at work & 44.3 & 44.8 & 0.805 & \\
\hline I have to do what I normally don't do & 60.4 & 59.2 & 0.575 & \\
\hline $\begin{array}{l}\text { I spend a lot more time on the internet searching for information } \\
\text { on COVID-19 }\end{array}$ & 43.3 & 42.7 & 0.78 & \\
\hline Too much news about COVID-19 on social media has affected me & 35.9 & 38.1 & 0.272 & \\
\hline
\end{tabular}

${ }^{*}$ Denotes $\mathrm{p}<0.01$.

ARI, Acute Respiratory Infection; HCWs, healthcare workers; PAPR, Powered Air Purifying Respirator; PPE, personal protective equipment.

were exacerbated by new-onset symptoms triggered by COVID- $19,{ }^{12}$ thus giving rise to a higher proportion than expected. Of note, this rate is still higher than that of $20 \%$ of HCWs as reported by Salehi et al in a recent systematic review and meta-analysis. ${ }^{13}$ Hence, the long intervening time between SARS and COVID-19 and prior experience as a SARS HCW do not appear to be protective.

Most SARS HCW respondents felt safer, better equipped, more confident about their healthcare leaders, and that their workload was being better managed. More of them also agreed with the various infection control/contact tracing measures and policies as well as being satisfied with official communications, likely due to a general perception of how things have generally improved from the SARS episode. However, at least half still reported fears of falling ill with COVID-19 and felt that their jobs put them at risk. More of them also doubted the effectiveness of protective equipment as compared with the nonSARS group. Memories of $42 \%$ of the local SARS patient population being HCWs and of losing one esteemed doctor colleague were likely still fresh. ${ }^{14}$ Like the SARS study, ${ }^{1}$ the large majority would continue their present jobs looking after infected patients; this was the same for the non-SARS HCWs. While it reflects an admirable work ethos from our HCWs, this could also reflect HCWs suppressing personal fears and emotions in a sacrificial attempt to provide patient care. This may be indicative of a trajectory towards the depletion of emotional resources and eventually fatigue and burnout, ${ }^{15}$ which are outcomes not measured by our study. Nevertheless, a possible target for intervention is to address the locus of control, since a sizeable proportion of both groups (more so for nonSARS HCWs) endorsed having little control over whether they get infected, and possession of an external locus of control has been identified as a vulnerability factor to psychological distress. ${ }^{16}$ It is possible that post-SARS strategies successfully addressed this or post-traumatic growth had spontaneously occurred in a number of SARS HCWs.
Various staff support systems were put in place after the SARS epidemic, and most participants were aware these resources were available to them. However, as the COVID-19 pandemic is more long-drawn and has more widespread impact across the healthcare system, we believe it is imperative to identify at-risk HCWs for targeted psychological outreach. Furthermore, despite the consensus of good morale at work, having adequate manpower, and adequate levels of PPE, high numbers still reported increased workplace conflict, more work stress and increased workload. Results of our logistic regression analyses showed that being more socially isolated (not living with family) was the most significant predictive factor for both SARS and non-SARS HCWs. This finding may be explained by the large numbers of foreign HCWs who were affected by the travel bans and prevented from being with their families in their countries of origin, as well as having to be housed in temporary accommodation due to distancing requirements placed on those living in communal housing. Even though these phenomena were only present during the current pandemic, we believe that these risk factors are not unique or specific to locale or pathogen-type and should be considered in future outbreak situations. Being exposed to confirmed/suspect cases daily was predictive of significant distress for nonSARS HCWs but not for SARS HCWs. This could reflect either a true protective effect of prior experience or a symptom of emotional numbing for SARS HCWs.

Considering that a quarter of the respondents experienced clinically significant trauma-related symptoms despite the existence of active HCW psychological support resources, we advocate for (1) the examination of current resources and their limitations in the current pandemic, (2) the modification of existing programmes to be more specifically trauma-informed in approach and (3) for more targeted and focused trauma-informed care for HCWs who have experienced repeated exposure. This calls for a resolve to acknowledge the presence and 
Table 4 Comparison of SARS and non-SARS HCWs who had IES scores in the moderate/severe range

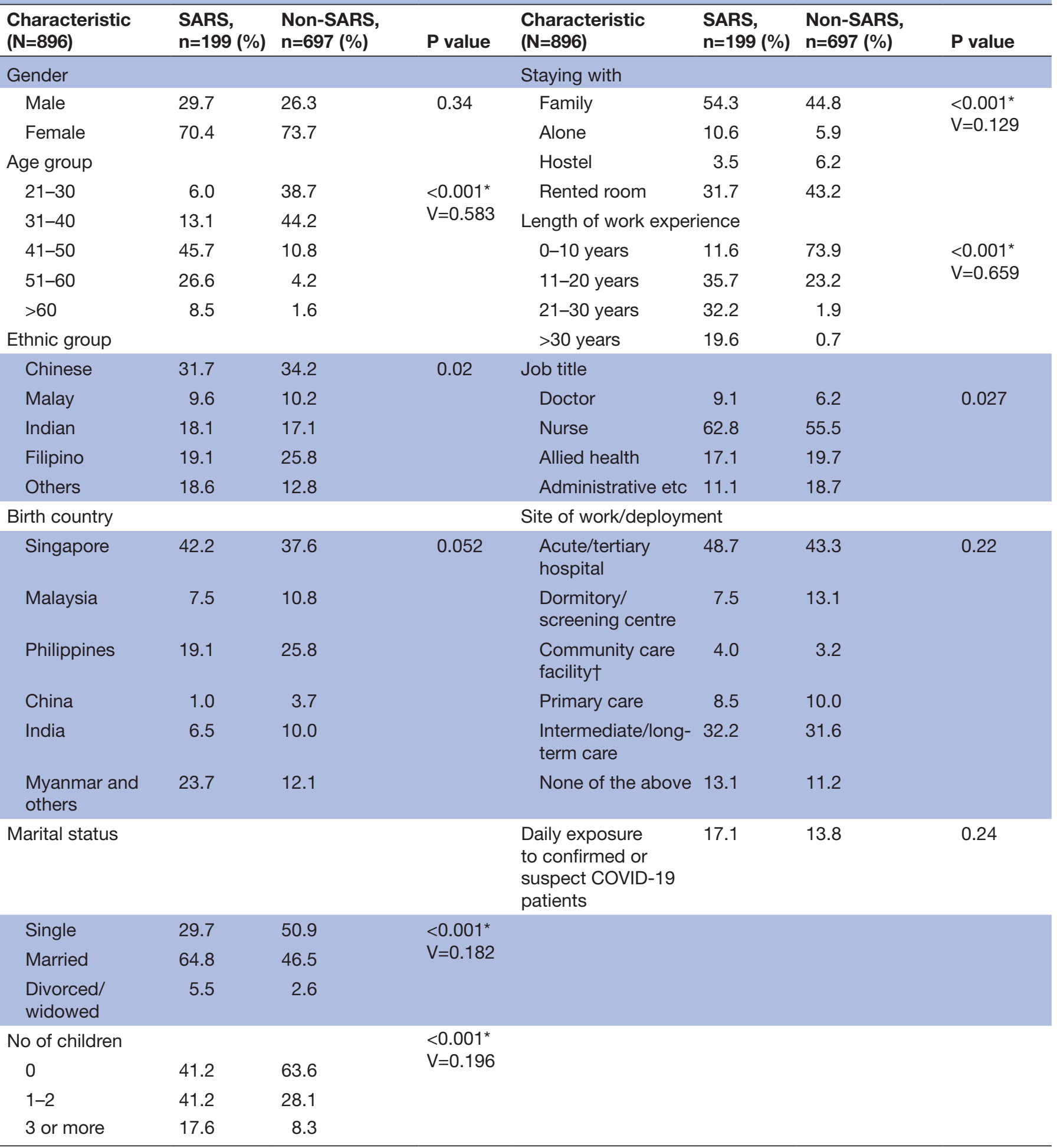

${ }^{*}$ Denotes $\mathrm{p}<0.01$.

†Community care facility- medically supervised care facilities for recovering COVID-19 patients till the completion of their infectious period. HCWs, healthcare workers; IES, Impact of Events Scale.

effects of vicarious trauma at the organisational level, ${ }^{17} 18$ and to transform the workplace climate so that HCWs feel safe, supported and well-supervised by a coach/mentor who has been trained in trauma-related care. ${ }^{19}$ This makes possible widespread training and education and the nomination of 'trauma-informed care champions' in different units who will be champions for the execution of informal networks for debrief and support. Additionally, suitable HCWs who have experienced SARS and identified to be coping well can be trained to leverage 
Table 5 Univariate logistic regression for predictors of moderate/severe IES score for SARS and non-SARS HCWs

\begin{tabular}{|c|c|c|c|c|c|c|}
\hline \multirow[b]{2}{*}{ Predictive factor } & \multicolumn{3}{|c|}{ SARS HCW } & \multicolumn{3}{|c|}{ Non-SARS HCW } \\
\hline & OR & $95 \% \mathrm{Cl}$ & $P$ value & OR & $95 \% \mathrm{Cl}$ & $P$ value \\
\hline \multicolumn{7}{|l|}{ Age group } \\
\hline $21-30$ & 1 & & & 1 & & \\
\hline $31-40$ & 0.38 & 0.15 to 0.97 & 0.042 & 0.85 & 0.70 to 1.04 & 0.114 \\
\hline $41-50$ & 0.36 & 0.15 to 0.84 & 0.018 & 0.66 & 0.49 to 0.89 & $0.006^{*}$ \\
\hline $51-60$ & 0.39 & 0.16 to 0.94 & 0.037 & 0.71 & 0.46 to 1.10 & 0.128 \\
\hline$>60$ & 0.19 & 0.07 to 0.51 & $0.001^{*}$ & 1.06 & 0.51 to 2.22 & 0.867 \\
\hline \multicolumn{7}{|l|}{ Ethnic group09† } \\
\hline Chinese & 1 & & & 1 & & \\
\hline Malay & 2.06 & 1.11 to 3.82 & $0.021^{*}$ & 1.50 & 1.10 to 2.05 & 0.011 \\
\hline Indian & 1.90 & 1.18 to 3.08 & $0.009^{*}$ & 1.61 & 1.24 to 2.09 & $<0.001^{*}$ \\
\hline Filipino & 2.53 & 1.55 to 4.12 & $<0.001^{*}$ & 2.12 & 1.68 to 2.69 & $<0.001^{*}$ \\
\hline Others & 2.43 & 1.52 to 3.88 & $<0.001^{*}$ & 1.56 & 1.04 to 2.56 & $0.003^{*}$ \\
\hline \multicolumn{7}{|l|}{ Birth country } \\
\hline Singapore & 1 & & & 1 & & \\
\hline Malaysia & 1.47 & 0.76 to 2.83 & 0.248 & 1.08 & 0.81 to 1.46 & 0.595 \\
\hline Philippines & 1.95 & 1.22 to 3.11 & $0.005^{\star}$ & 1.89 & 1.50 to 2.39 & $<0.001^{*}$ \\
\hline China & 0.28 & 0.06 to 1.20 & 0.086 & 1.00 & 0.63 to 1.59 & 0.993 \\
\hline India & 1.14 & 0.58 to 2.24 & 0.703 & 1.56 & 1.13 to 2.15 & $0.006^{*}$ \\
\hline Myanmar & 2.00 & 1.26 to 3.19 & $0.004^{*}$ & 1.20 & 0.86 to 1.67 & 0.275 \\
\hline Others & 5.33 & 1.70 to 16.73 & $0.004^{*}$ & 1.22 & 0.75 to 2.01 & 0.413 \\
\hline \multicolumn{7}{|l|}{ Marital status } \\
\hline Single & 1 & & & 1 & & \\
\hline Married & 0.65 & 0.45 to 0.95 & 0.024 & 0.90 & 0.76 to 1.08 & 0.267 \\
\hline Divorced/widowed & 1.51 & 0.57 to 4.05 & 0.404 & 0.93 & 0.52 to 1.65 & 0.806 \\
\hline \multicolumn{7}{|l|}{ No of children } \\
\hline 0 & 1 & & & 1 & & \\
\hline $1-2$ & 0.66 & 0.46 to 0.95 & 0.027 & 0.79 & 0.65 to 0.96 & 0.018 \\
\hline 3 or more & 0.85 & 0.53 to 1.37 & 0.513 & 0.81 & 0.59 to 1.11 & 0.195 \\
\hline \multicolumn{7}{|l|}{ Staying with†‡ } \\
\hline Family & 1 & & & 1 & & \\
\hline Alone & 1.88 & 1.06 to 3.32 & 0.030 & 1.54 & 1.04 to 2.27 & 0.03 \\
\hline In hostel & 2.78 & 1.01 to 7.64 & 0.047 & 3.06 & 2.00 to 4.70 & $<0.001^{*}$ \\
\hline In rented room/apartment & 2.96 & 1.99 to 4.40 & $<0.001^{*}$ & 1.95 & 1.61 to 2.36 & $<0.001^{*}$ \\
\hline \multicolumn{7}{|l|}{ Length of work experience } \\
\hline $0-10$ years & 1 & & & 1 & & \\
\hline $11-20$ years & 0.41 & 0.22 to 0.76 & $0.005^{*}$ & 0.73 & 0.59 to 0.89 & $0.002^{*}$ \\
\hline $21-30$ years & 0.53 & 0.29 to 1.00 & 0.049 & 0.72 & 0.38 to 1.35 & 0.307 \\
\hline$>30$ years & 0.37 & 0.19 to 0.72 & $0.003^{*}$ & 1.45 & 0.47 to 4.47 & 0.513 \\
\hline \multicolumn{7}{|c|}{ Daily exposure to confirmed/suspect casesł } \\
\hline No & 1 & & & 1 & & \\
\hline Yes & 1.28 & 0.82 to 1.20 & 0.283 & 1.48 & 1.14 to 1.94 & $0.004^{*}$ \\
\hline
\end{tabular}

${ }^{*}$ Denotes $\mathrm{p}<0.01$.

†Denotes variable remains significant after multivariate logistic regression for non-SARS HCW group. ‡Denotes variable remains significant after multivariate logistic regression for SARS HCW group. HCW, healthcare worker; IMS, Impact of Events Scale. 
on their experience to provide trauma-informed care to affected colleagues. Nevertheless, the real challenge is in the actualisation of protocols that require interpersonal interactions in the face of adhering to infection control guidelines and manpower conservation measures such as the freezing of vacation days.

We acknowledge that a major limitation of our study is that we chose not to use the IES-Revised (IES-R) scale or other scales that measured depression/anxiety levels, personality and resilience factors, and that reduces the comparability of our results with those from other studies. ${ }^{20}$ In view of the unique nature of our healthcare workforce having experienced the similar SARS crisis, we intentionally adapted our questionnaire from a similar one used in 2003 during the SARS epidemic to glean insight and make general comparisons between it and the current pandemic. We believed that performing a cross-sectional measurement using general psychiatric measures would not add novel value beyond existing studies on the mental health of HCWs; hence, we avoided this approach. Another limitation of this online survey methodology is that it may not have reached those who did not have institutional email addresses or who were not adequately IT-savvy, thus introducing sample bias and affecting the generalisability of the results. We had proposed having study team members to conduct in-person outreach to departments such as portering, housekeeping, catering and security, however, it was vetoed as it contravened infection control policies. Hence, we were also not able to report the response/nonresponse rate and examine the characteristics of those who did not respond to the survey. As the survey was anonymous, we were also unable to reidentify respondents in order to clarify unclear responses. Respondents also did not have opportunity to clarify the items they did not understand and thus causing opportunities for erroneous responses. The final sample size may also have been limited by the lack of incentive for completing the survey due to lack of funding. Regrettably, we were unable to reach a similar sample size to the 2003 SARS study.

We designed our questionnaire to include novel openended questions for survey respondents to upload photos, videos, journal entries or free-text responses on how they have been coping during the pandemic. These data are being analysed qualitatively and will be reported elsewhere. Each site-principal investigator was also provided with the site-specific dataset of both the quantitative and qualitative data for use in their own sites. It was beyond the scope of this paper to report and discuss results by site or healthcare setting. The study team hopes to eventually combine the results from the quantitative and qualitative sections and make useful recommendations to our healthcare leadership for the ongoing psychological protection of our healthcare workforce. Future directions should focus on the effectiveness of such strategies and the longitudinal follow-up of the well-being of HCWs considering the protracted nature of the current pandemic which was still unknown at the time the study was conceptualised and conducted.

\section{Author affiliations}

${ }^{1}$ Psychiatry, Tan Tock Seng Hospital, Singapore

${ }^{2}$ Adult Psychiatry, Institute of Mental Health, Singapore

${ }^{3}$ General Psychiatry, Institute of Mental Health, Singapore

${ }^{4}$ Psychological Medicine, Khoo Teck Puat Hospital, Singapore

${ }^{5}$ National Skin Centre, Singapore

${ }^{6}$ Anaesthesia, Ng Teng Fong General Hospital, Singapore

${ }^{7}$ Clinical Research Unit, National Healthcare Group Polyclinics, Singapore

${ }^{8}$ Medical Psychiatry, Woodlands Health Campus, Singapore

${ }^{9}$ Palliative Medicine, Woodlands Health Campus, Singapore

${ }^{10}$ Dover Park Hospice, Singapore

${ }^{11} \mathrm{National}$ Centre for Infectious Diseases, Singapore

${ }^{12}$ Infectious Diseases, Tan Tock Seng Hospital, Singapore

${ }^{13}$ Department of Clinical Epidemiology, Tan Tock Seng Hospital, Singapore

${ }^{14}$ Lee Kong Chian School of Medicine, Nanyang Technological University, Singapore

Acknowledgements We thank Assistant Professor Zoe Jane-Lara Hildon from National University of Singapore for her contributions to the qualitative aspects of the study, Professor David Koh from Universiti Brunei Darussalam for granting permission to adapt the survey used in the 2003 SARS study, and Miss Olivia Harmony Chan Yeong from Tan Tock Seng Hospital for her contributions to the editing and formatting of the manuscript. We also thank AIC for their assistance in disseminating the survey to their ILTC partners.

Contributors LGC: overall principal investigator, study design, coordination of all study sites, data collection, data analysis and interpretation, drafting and finalising manuscript, guarantor for the study accepting full responsibility for the work and the conduct of the study, have access to the data, and controlled the decision to publish.PLLT: Study design, data analysis and interpretation, finalising manuscript. KS: site principal investigator (IMH), data collection, data interpretation, finalising manuscript. MYT: site co-principal investigator (IMH), data collection, data interpretation and finalising manuscript. KHG:site principal investigator (KTPH), data collection, data interpretation and finalising manuscript. PQS: site principal investigator (National Skin Centre), data collection, data interpretation and finalising manuscript. AKHT: site principal investigator ( $\mathrm{Ng}$ Teng Fong General Hospital), data collection, data interpretation, finalising manuscript. ESL: site principal investigator (National Healthcare Group Polyclinics), study design, data collection, data interpretation, finalising manuscript. SYT: site co-principal investigator (National Healthcare Group Polyclinics), study design, data collection, data interpretation, finalising manuscript. WPL: site principal investigator (Woodlands Health Campus), data collection, data interpretation, finalising manuscript. CHA: site co-principal investigator (Dover Park Hospice), data collection, data interpretation, finalising manuscript. YZG: site principal investigator (Dover Park Hospice), data collection, data interpretation, finalising manuscript. SS: site principal investigator (National Centre for Infectious Diseases), study design, data collection, data interpretation, finalising manuscript. AC: study design, data interpretation and finalising manuscript. All gave final approval and agree to be accountable for all aspects of work ensuring integrity and accuracy.

Funding The authors have not declared a specific grant for this research from any funding agency in the public, commercial or not-for-profit sectors.

\section{Competing interests None declared.}

Patient consent for publication Not applicable.

Ethics approval National Healthcare Group Domain Specific Review Boards. Approval number DSRB 2020/00385.

Provenance and peer review Not commissioned; externally peer reviewed.

Data availability statement All data relevant to the study are included in the article or uploaded as online supplemental information. All deidentified participant data are from healthcare workers in Singapore. The data have been included as supplementary material along with this manuscript. The data can be reused only under direct permission from the PI. The PI can be contacted at lai_gwen_chan@ ttsh.com.sg, +6563577841 .

Supplemental material This content has been supplied by the author(s). It has not been vetted by BMJ Publishing Group Limited (BMJ) and may not have been peer-reviewed. Any opinions or recommendations discussed are solely those of the author(s) and are not endorsed by BMJ. BMJ disclaims all liability and responsibility arising from any reliance placed on the content. Where the content includes any translated material, BMJ does not warrant the accuracy and reliability of the translations (including but not limited to local regulations, clinical guidelines, terminology, drug names and drug dosages), and is not responsible 
for any error and/or omissions arising from translation and adaptation or otherwise.

Open access This is an open access article distributed in accordance with the Creative Commons Attribution Non Commercial (CC BY-NC 4.0) license, which permits others to distribute, remix, adapt, build upon this work non-commercially, and license their derivative works on different terms, provided the original work is properly cited, appropriate credit is given, any changes made indicated, and the use is non-commercial. See: http://creativecommons.org/licenses/by-nc/4.0/.

\section{ORCID iDs}

Lai Gwen Chan http://orcid.org/0000-0003-3553-1525

Kang Sim http://orcid.org/0000-0003-3209-9626

Eng Sing Lee http://orcid.org/0000-0003-4963-535X

\section{REFERENCES}

1 Koh D, Lim MK, Chia SE, et al. Risk perception and impact of severe acute respiratory syndrome (SARS) on work and personal lives of healthcare workers in Singapore: what can we learn? Med Care 2005;43:676-82.

2 Chan AOM, Huak CY. Psychological impact of the 2003 severe acute respiratory syndrome outbreak on health care workers in a medium size regional General Hospital in Singapore. Occup Med 2004;54:190-6.

3 McAlonan GM, Lee AM, Cheung V, et al. Immediate and sustained psychological impact of an emerging infectious disease outbreak on health care workers. Can J Psychiatry 2007;52:241-7.

4 Maunder RG, Lancee WJ, Rourke S, et al. Factors associated with the psychological impact of severe acute respiratory syndrome on nurses and other Hospital workers in Toronto. Psychosom Med 2004;66:938-42.

5 Maunder R, Lancee W, Balderson K, et al. Long-Term psychological and occupational effects of providing Hospital healthcare during SARS outbreak. Emerg Infect Dis 2006;12:1924-32.

6 Bai Y, Lin C-C, Lin C-Y, et al. Survey of stress reactions among health care workers involved with the SARS outbreak. Psychiatr Serv 2004;55:1055-7.

7 Wu P, Fang Y, Guan Z, et al. The psychological impact of the SARS epidemic on hospital employees in China: exposure, risk perception, and altruistic acceptance of risk. Can J Psychiatry 2009;54:302-11.
8 Spoorthy MS, Pratapa SK, Mahant S. Mental health problems faced by healthcare workers due to the COVID-19 pandemic-A review. Asian J Psychiatr 2020;51:102119.

9 Baker AJ. Singapore's circuit breaker and beyond: Timeline of the COVID-19 reality. Secondary Singapore's circuit breaker and beyond: Timeline of the COVID-19 reality 15 June 2020, 2020. Available: https://www.channelnewsasia.com/news/singapore/covid-19-circuitbreaker-chronicles-charting-evolution-12779048

10 Hutchings E, Devilly GJ. Impact of event scale (IES). secondary impact of event scale (IES). Available: http://www.clintools.com/ victims/resources/assessment/ptsd/ies.html

11 Dominguez-Gomez E, Rutledge DN. Prevalence of secondary traumatic stress among emergency nurses. J Emerg Nurs 2009;35:199-204.

12 Lancee WJ, Maunder RG, Goldbloom DS, et al. Prevalence of psychiatric disorders among Toronto Hospital workers one to two years after the SARS outbreak. Psychiatr Serv 2008;59:91-5.

13 Salehi M, Amanat M, Mohammadi M, et al. The prevalence of post-traumatic stress disorder related symptoms in coronavirus outbreaks: a systematic-review and meta-analysis. J Affect Disord 2021;282:527-38.

$14 \mathrm{MoH}$ S. Special feature: severe acute respiratory syndrome (SARS). communicable diseases surveillance in Singapore 2003, 2003.

15 Caldas MP, Ostermeier K, Cooper D. When helping hurts: COVID-19 critical incident involvement and resource depletion in health care workers. J Appl Psychol 2021;106:29-47.

16 Conversano C, Marchi L, Miniati M. Psychological distress among healthcare professionals involved in the covid-19 emergency: vulnerability and resilience factors. Clinical Neuropsychiatry 2020;17:94-6.

17 Keesler JM. Promoting satisfaction and reducing fatigue: understanding the impact of trauma-informed organizational culture on psychological wellness among direct service providers. J Appl Res Intellect Disabil 2020;33:939-49.

18 Ashley-Binge S, Cousins C. Individual and organisational practices addressing social workers' experiences of vicarious trauma. Practice 2020;32:191-207.

19 Palm KM, Polusny MA, Follette VM. Vicarious traumatization: potential hazards and interventions for disaster and trauma workers. Prehosp Disaster Med 2004;19:73-8.

20 Mosolova E, Sosin D, Mosolov S. Stress, anxiety, depression and burnout in frontline health care workers during COVID-19 pandemic: a brief systematic review and new data from Russia 2021. 